\title{
Oryx and Crake
}

\section{Erhard Taverna}

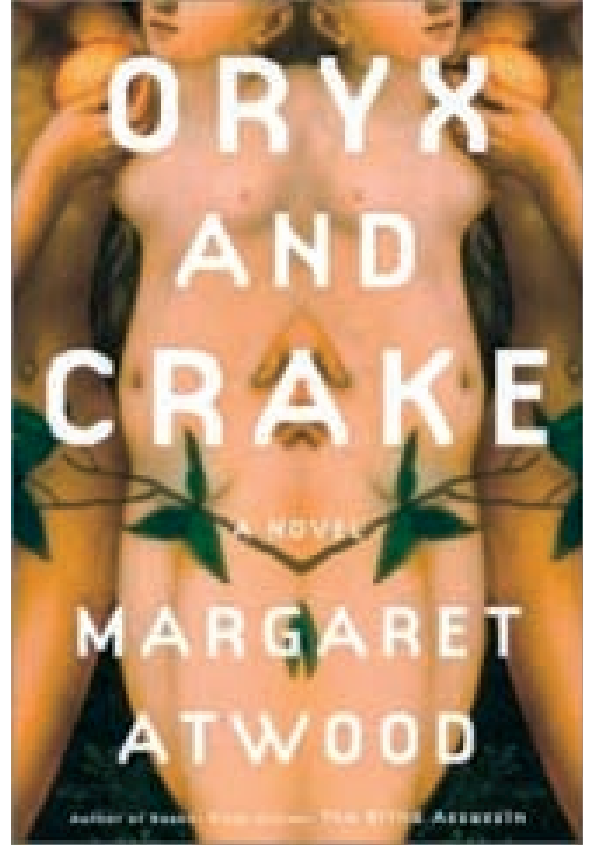

Einsatzkonzepte bei B-Ereignissen gehören zum Inventar der Krisenstäbe. Seit Jahren werden Katastrophenpläne für die erwartete Grippepandemie ausgebrütet, Interventionspläne angepasst, Impfstrategien ausgeheckt, Prioritätenlisten erstellt, Best- und Worst-Case-Szenarien durchgeprobt. Anschläge mit Milzbrandsporen und Ausbrüche von Ebola und SARS drohen wie Wetterleuchten an der epidemiologischen Front. Als wäre dies nicht genug, weiss ein jeder, dass unzählige Experten in geheimen Labors der Militärs und Geheimdienste die bakteriologische Kriegsführung unter dem Vorwand ihrer Bekämpfung weiterentwickeln.

Wenn eine kanadische Schriftstellerin wie Margaret Atwood mit ihren Mitteln eine nächste Zukunft auf diesem unsicheren Terrain erforscht, dann ist das mehr als ein weiterer Beitrag zu den vielen Horrorvisionen, die es zu diesem Thema bereits gibt. Sie hat schon mehrmals einen guten Riecher für politische Entwicklungen bewiesen, das letzte Mal vor zehn Jahren mit ihrem Roman «The Handmaid's Tale». Sie beschrieb damals, aus der Sicht einer Gebärsklavin, ein von Bürgerkriegen zerrissenes Nordamerika, in dem religiöse Fundamentalisten ein Terrorregime errichteten. Der Abtreibung für schuldig befundene Ärzte werden dort erschossen und als Leichen öffentlich zur Schau stellt. Science-fiction ist nicht ihre Sache im Sinne technikschwelgerischer Utopien. Sie versucht mit sehr viel Vorstellungsvermögen, gegenwärtige gesellschaftliche Tendenzen in die nächste Zukunft zu verlängern.

Der neue Roman, «Oryx and Crake», handelt auf mehreren Erzählebenen. Ein Überlebender, der seiner weissen Haare wegen Snowman heisst, berichtet in langen inneren Monologen, Träumen und Halluzinationen von der Vergangenheit. Er selber hat als Jimmy noch erlebt, wie in England BSE-Rinder verbrannt wurden. Crake ist der Codename für seinen besten Schulfreund, der als Genforscher eine Spitzenposition erklimmt. Oryx nennen sie ihre gemeinsame Freundin, eine asiatische Schönheit mit einer schlimmen Vergangenheit, die am Ende erneut, aber auf eine andere Art missbraucht wird.

Die Freunde leben in komfortablen, von der Umwelt abgeschirmten Siedlungen, die untereinander mit schnellen, gepanzerten Privatzügen verbunden sind. Dort sorgen die konkurrenzierenden Pharmaunternehmen für allen Komfort, für gute Schulen, keimfreies Wasser, effiziente Impfprogramme und viel elektronische Unterhaltung. Ausserhalb dieser «gated cities» leben die Unterprivilegierten in überfüllten Städten mit wachsender Kriminalität und zunehmenden Umweltschäden. Crake und Jimmy vertreiben sich die Freizeit mit einer Zukunftsvariante gegenwärtiger Videogames, darunter mit einem verhängnisvollen Programm, das sich «Extinctathon» nennt. Eine Figur namens «Mad Adam» - «mad», weil er im Gegensatz zu Adam nur die toten Tiere nennt - ist eine der vielen verhängnisvollen Figuren, die virtuos erfunden, mit Sicherheit die aktuelle Unterhaltungsindustrie beflügeln werden. Jimmy, der mathematisch weniger begabte, studiert angewandte Rhetorik. Er digitalisiert Bibliotheken, bevor das letzte Buch vernichtet wird, und erstellt ein Lexikon nicht mehr geläufiger Ausdrücke. Crake schafft es an die Spitze eines renommierten Forschungslabors, wo ihm die Geschäftsleitung für einen enorme Gewinne versprechenden Blockbuster alle Privilegien und Kompetenzen zugesteht. Während alle alten Arten verschwinden, entstehen überall industriell gezüchtete Hybride von Tieren und Pflanzen. Für das Spleissen der 
Gene und die Erfindung immer neuer Proteine gibt es schon lange keine Barrieren mehr. Kurz vor der Endphase seines geheimgehaltenen Planes holt Crake seinen Freund zu sich in das Paradies genannte Labor. Hinter Schleusen gesichert, erlebt Jimmy an allen Monitoren den Ausbruch einer tödlichen, globalen Epidemie, die, gut geplant, zeitgleich auf allen Kontinenten losbricht. Als Einsiedler, der die Nacht auf Bäumen verbringt, wird er von gentechnisch gezüchteten «Wilden», dem Vermächtnis Crakes für die Zeit danach, als dessen Prophet verehrt und ernährt.
Die Idee, dass ein Unternehmen namens Meridian Bioscience in Cincinnati im September 2004 an 6400 Labors versehentlich einen tödlichen Grippevirus versenden würde, hätte von Margaret Atwood sein können.

- Margaret Atwood. Oryx and Crake. Paperback. London: Virago Press; 2003. Seit 2005 auch in deutscher Übersetzung «Oryx und Crake» beim Berlin Verlag (gebunden) oder Berliner Taschenbuch Verlag (Paperback) erhältlich.

\title{
Robotiser ou (ré)humaniser la médecine?
}

\author{
R. M. Jolidon
}

Correspondance:

Dr René-Marc Jolidon Rue des Remparts 18 CH-1400 Yverdon-les-Bains
La médecine n'est plus un art. L'art, en soi, ne doit pas être rentable économiquement, c'est-àdire prêt à fournir un service au prix le plus concurrentiel possible. De même, elle ne peut se prévaloir d'un humanisme que si la notion de rentabilité est définie par l'apport d'un bénéfice (non pécuniaire) satisfaisant pour le bien-être des membres de la société. Or en pratique, on ne parle plus que de coût, de budget, de déficits. Le médecin ne devient qu'un prestataire de service, un maillon comptable soumis à la logique économiste, de manière dogmatique et dictatoriale.

Et pourtant je suis heureux ... heureux de constater que d'éminents cerveaux, des experts en procédures et processus de tout poil triment et courbent l'échine en nous proposant leurs décisions dans un des derniers numéros du BMS [1] ...: la commission paritaire d'interprétation du TARMED a la chance de s'assurer les services de grands esprits, qui manient la vanité (de vanitas) avec un certain brio. Ah! Le TARMED ... cette bible néo-hippocrato-libérale, ce monument des sciences de l'économie (evidence-based?) appliqué aux sciences sociales, biologiques et médicales. Elles nous manquaient ces mises au point et ces explications très travaillées bien qu'insuffisantes pour rendre ce pensum plus comestible!

La position 03013 nous rappelle notre rapport avec le temps, la vie n'étant qu'un bref passage sur cette planète: juste le temps compté pour donner votre sueur et votre solde à la banque, à l'Etat ou à leurs complices. C'est vrai, le temps est si précieux quand il sonne et tré- buche qu'il est indispensable de ne pas oublier l'importance de la 301 nième seconde. Comme il est doux et utile de se pencher sur le nombre de caractères de nos rapports qui permet de cadrer nos envies d'écriture et nous faire oublier l'angoisse de la page blanche. Comme il est «régénérateur» pour un universitaire, diplômé d'Etat, et dans la force de l'âge, de se laisser baigner par la position 04.0900 ou 04.0920 qui nous aide à interpréter «que la profondeur mesurée à angle droit depuis la surface du corps est déterminante pour définir à quelle profondeur se situe le corps étranger».

Alors là, Messieurs, chapeau bas, vous avez fait très fort dans la volonté de simplifier les choses. Et pourquoi ne pas accorder quelques heures supplémentaires de réflexion, tout aussi profonde, à ces âmes bien faites et fleurant bon l'épicerie pour déterminer si ce mode d'évaluation concerne tous les corps étrangers et dans n'importe quel orifice. Dans le même ordre d'idée («la grande médecine s'évalue dans les petits gestes», disait un de mes patrons), faudra-t-il tenir compte du nombre de poils à raser sur le thorax du patient-client lors d'un ECG pour tarifer de manière adéquate? Du nombre de boutons dans une varicelle? Du poids ou de la longueur du bouchon de cérumen dans le conduit auditif? Une médecine à la Pierre Dac ... Plus ou moins drôle, cantonnée à sonder l'absurde pour justifier ses revenus ...

La justice doit s'exercer dans la sérénité ... tout le monde en convient. La médecine n'a plus 
ce privilège. Prise en otage par les assureurs, les réalités économiques des politiques, des boîtes pharmaceutiques, par les exigences des patients de moins en moins instruits par des journalistes dénués d'éthique, cernée et contrainte par des mesures de secondes, de centimètres et de nombres de caractères, notre profession souffre dans son indépendance et dans sa relation humaine. Ces nombreux paramètres sont moins destinés à la rendre science exacte qu'à nous rappeler que la vie a un coût et que celle-ci rime avec roupies. L'écoute de son prochain et l'étude de son problème, le partage des connaissances et d'empathie, le devoir d'accompagnement sont mis à (la)Mal par une ribambelle de dépendances sournoises imposées de toutes parts, y compris de nos rangs. La médecine a changé de mission et le missionnaire n'a toujours pas de position dans le TARMED.

Depuis la chute de certaines idéologies, on tente de nous faire croire que les dictatures sont mortes: que nenni, c'est le profil qui change ... le tout à l'économie, ils sont là, les nouveaux despotes ... dans l'économie saupoudrée d'informatique et de statistiques peu vérifiables, opaques et pas toujours interprétables. Heureusement ... habemus diazépam pour supporter tout ça, diront certains. Et tout est transitoire ... il suffit d'un peu de patience...

Tout système est perfectible. Cela demande questionnements, réflexions individuelles et communes. Est-ce que la médecine rapporte (et peut-on dire combien) ou ne fait-elle que coûter? La santé a-t-elle perdu sa dimension universelle, un bien public mondial, pour devenir un moyen au service de la production? Est-ce que l'intervention des Etats ne devrait pas se faire davantage dans le contrôle des prix des médicaments et du matériel médical que dans notre pratique jugée dispendieuse? Les effets de manches tarifaires ne sont-ils pas un leurre pour justifier nos profits sans changer ni la forme ni le fond de la problématique des coûts de la santé? Quel poids (ou quel rôle social) a le médecin dans la définition des priorités en matière de santé dans notre société? Quelles sont-elles? Est-ce que la médecine peut répondre à toutes les attentes par la course en avant dans les techniques? Dans ce monde «inégalitariste», consumériste et à la spiritualité déficiente, le médecin de premier recours ne devient-il pas le pasteur du dernier secours?

Quelques soient les questions, dans la recherche des réponses, il faut savoir dire non sans artifice à ce qui paraît injuste et futile; le ciment de la réflexion doit être fait de bon sens ... du bon sens à gogo ... et le cultiver intensément dans le sens de l'humain et de son environnement. D'ailleurs, ça se tarifie comment, le bon sens?

\section{Référence}

1 TARMED Suisse. Décisions de la Commission paritaire d'interprétation. Bull Méd Suisses 2005; 86(16):931-3. 